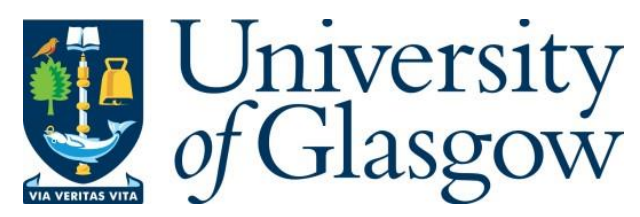

Goldberg, G. R., Boldin, A., Andersson, S. M.L., Ivanov, P., Ozaki, N., Taylor, R. J.E., Childs, D. T.D., Groom, K. M., Kennedy, K. L. and Hogg, R. A. (2017) Gallium nitride superluminescent light emitting diodes for optical coherence tomography applications. IEEE Journal of Selected Topics in Quantum Electronics, 23(6), pp. 1-11.

There may be differences between this version and the published version. You are advised to consult the publisher's version if you wish to cite from it.

http://eprints.gla.ac.uk/147516/

Deposited on: 7 September 2017

Enlighten - Research publications by members of the University of Glasgow http://eprints.gla.ac.uk 


\title{
Gallium Nitride Superluminescent Light Emitting Diodes for Optical Coherence Tomography Applications
}

\author{
Graham R. Goldberg, Aleksandr Boldin, Sophia M. L. Andersson, Pavlo Ivanov, Nobuhiko Ozaki, \\ Richard J. E. Taylor, David T. D. Childs, Member, IEEE, Kristian M. Groom, Member, IEEE, Kenneth L. Kennedy, \\ and Richard A. Hogg
}

\begin{abstract}
Optical coherence tomography (OCT) exploits the coherent properties of light to permit noninvasive and in situ imaging of biological tissues. By expanding the range of OCT light sources from the traditional telecoms wavelengths to include $\sim 400$ nm gallium nitride (GaN) based superluminescent light emitting diodes (SLEDs) subcellular axial and lateral resolution could be achieved, provided enhanced bandwidth is also achieved. Due to the focus on high-power applications for GaN SLEDs, there has been limited work on increasing the source bandwidth. In this paper, we demonstrate for the first time a $\sim 400 \mathrm{~nm}$ GaN SLED with $>10 \mathrm{~nm}$ bandwidth employed within an OCT system, where an axial resolution of $\sim 7 \mu \mathrm{m}$ is achieved. Bespoke GaN SLEDs suggest that $<4 \mu \mathrm{m}$ axial resolution imaging is imminent for short wavelength devices.
\end{abstract}

Index Terms-Axial resolution, broad bandwidth, gallium nitride superluminescent light emitting diodes, optical coherence tomography.

\section{INTRODUCTION}

G ALLIUM nitride $(\mathrm{GaN})$ has been the recent subject of extensive research with the first viable GaN light emitting diodes (LEDs) and lasers reported by Nakamura et al. in 1991 and 1996, respectively [1], [2]. Marked improvements in the crystal quality and III-nitride growth and fabrication [3] have

The work of G. R. Goldberg and S. M. L. Andersson was supported in part by The Engineering and Physical Sciences Research Council (EPSRC) and The University of Glasgow. (Corresponding author: Graham R. Goldberg.)

G. R. Goldberg, S. M. L. Andersson, P. Ivanov, D. T. D. Childs, and R. A. Hogg are with the School of Engineering, The University of Glasgow, Glasgow G12 8LT, U.K. (e-mail: g.goldberg.1 @ research.gla.ac.uk; s.andersson.1 @ research.gla.ac.uk; pavlo.ivanov@glasgow.ac.uk; David.Childs@glasgow.ac. uk; richard.hogg@glasgow.ac.uk).

A. Boldin, K. M. Groom, and K. L. Kennedy are with the Department of Electronic and Electrical Engineering, The University of Sheffield, Sheffield S3 7HQ, U.K. (e-mail: aboldin1@ @ sheffield.ac.uk; k.m.groom@sheffield.ac.uk; k.kennedy@sheffield.ac.uk).

N. Ozaki is with the Faculty of Systems Engineering, Wakayama University, Wakayama 640-8510, Japan (e-mail: ozaki@sys.wakayama-u.ac.jp).

R. J. E. Taylor is with the Department of Electrical Engineering and Information Systems, The University of Tokyo, Tokyo 113-8656, Japan (e-mail: richard.taylor@hotaka.t.u-tokyo.ac.jp).

Color versions of one or more of the figures in this paper are available online at http://ieeexplore.iee.org.

Digital Object Identifier provided higher powers and longer lifetime operation [4]-[6]; enabling the use of GaN devices in solid state lighting [7] and high-density optical media [8].

Superluminescent light emitting diodes (SLEDs) are waveguided devices where feedback is suppressed [9] to allow high amplified spontaneous emission powers. SLEDs are typically several times longer than their laser counterparts, with output powers often limited by the onset of lasing. They offer a low divergence point source with potential for broad spectral bandwidth and high powers. The first GaN SLED was described by Feltin et al. where $4.6 \mathrm{~nm}$ bandwidth and an output power of 10 $\mathrm{mW}$ were achieved under pulsed operation [10].

Realization of practical GaN SLEDs has diversified the application range for short wavelength $(\sim 400 \mathrm{~nm})$ optical devices to include pico-projectors [11]. However, because these sources require high output power and only moderate bandwidth, GaN SLED development has thus far focused on enhancing device tolerance to high current density; through improved thermal management [12], and feedback suppression [9]. A number of reported GaN SLEDs have high reflectivity coatings applied to their rear facets [13] effectively doubling cavity length for higher efficiency and reduced chip cost. Recently, output powers of $>200 \mathrm{~mW}$ under continuous wave have been achieved [14], [15]. However, to date there has been limited effort towards broad bandwidth GaN devices.

Optical coherence tomography (OCT) utilizes coherent light sources for non-invasive and in situ imaging of biological tissues, in particular the eye and skin. Since Huang et al. first reported OCT in 1991 [16], imaging speed and axial resolution have increased, with the latter limited by central wavelength and bandwidth of the light source [17]. Optical coherence microscopy (OCM) combines OCT and confocal microscopy [18], replacing the low numerical aperture (NA) focusing lens used in OCT with a high NA objective lens in order to image at a fixed, and greater, depth within the sample, improving the depth of field contrast limitations of OCT systems [19].

Demand for increased axial resolution has led to attempts to extend the traditional wavelengths used for OCT and OCM beyond 800 and $1300 \mathrm{~nm}$; with super-continuum lasers [20] and non-linear fiber-based light sources [21] giving access to shorter wavelengths. It has recently been demonstrated that in vivo OCT imaging at $\sim 400 \mathrm{~nm}$ is not only possible on human skin, but that 
ultraviolet light is able propagate to the dermal layer [22], providing clinicians with additional diagnostic images. However, complex systems are required to make use of these light sources [23]. Sub-cellular axial resolution can be achieved if broader bandwidth short wavelength SLEDs can be realized [24], in addition to an enhanced lateral resolution; highlighting a clear opportunity for broad bandwidth SLEDs emitting at $\sim 400 \mathrm{~nm}$ within both OCT and OCM systems.

Although various configurations of SLED design and cavity suppression have been proposed [9], limited work has been conducted on the practical use of absorbing section(s) in $\mathrm{GaN}$ SLEDs [25]. An absorbing section is electrically isolated from the light emitting section of a waveguide but is optically connected, with the absorbing section(s) ideally operated in loss. Optical loss within the absorber section(s) is significant, suppressing the onset of lasing. The absorber can be operated in open circuit $(\mathrm{O} / \mathrm{C})$, short circuit $(\mathrm{S} / \mathrm{C})$, or reverse biased mode. Kwong et al., demonstrated that by using an $\mathrm{O} / \mathrm{C}$ absorber in GaAs SLEDs the lasing threshold, $J_{\text {th }}$, could be increased, suppressing lasing at lower currents [26]. However, at higher currents the $\mathrm{O} / \mathrm{C}$ absorber became optically pumped by the light emission section in a process termed "burning through" and lasing was observed. By grounding the absorber, it was shown that $J_{\text {th }}$ was further increased; attributed to the path to ground preventing carriers from accumulating in the absorbing section. This is discussed in detail in Section IV.

Laterally patterned substrates have realized bandwidths of $6.1 \mathrm{~nm}$ under high current densities [27]. By varying quantum well $(\mathrm{QW})$ indium composition between the rear and front facet, the range of emission wavelengths is increased along the device, and light reabsorption is reduced, giving rise to an increased spectral bandwidth [28].

In this paper we explore the role of the full-width halfmaximum (FWHM) or $-3 \mathrm{~dB}$ bandwidth, central wavelength and numerical aperture (NA) on the axial and lateral resolution of OCT systems, highlighting the opportunities for subcellular resolution imaging using broadband GaN based light sources. We show that a commercial GaN laser can be modified to produce a SLED, and commission a $\sim 400 \mathrm{~nm}$ time-domain OCT system. Different methods to determine OCT system axial resolution are compared and metrology measurements are performed on a transparent sample. Finally, we discuss the effect of piezoelectric and spontaneous polarization in $\mathrm{GaN}$ devices and show experimentally, on bespoke GaN SLEDs, that absorber bias conditions play a critical role in the bandwidth of $\mathrm{GaN}$ SLEDs. Such devices are shown to lend themselves to $\sim 4 \mu \mathrm{m}$ axial resolution OCT imaging.

\section{THEORETICAL RESOLUTION OF OCT SySTEMS}

The axial resolution of a time-domain OCT system is determined by the coherence length of the optical source [17]-[23]. Whilst for a real source the deduction of the theoretical resolution is often obtained through the calculation of the point spread function (PSF) [29], [30], for an ideal broadband source with a Gaussian spectrum, it can be shown that

$$
l_{c}=\frac{2 \ln (2)}{\pi} \cdot \frac{\lambda_{0}^{2}}{\Delta \lambda}
$$

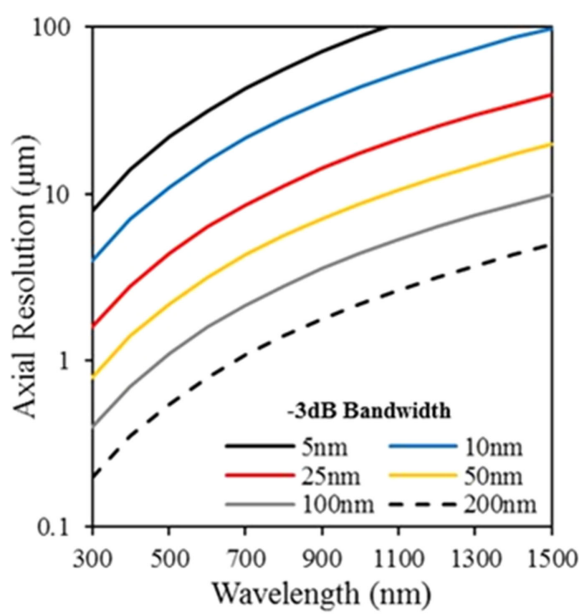

(a)

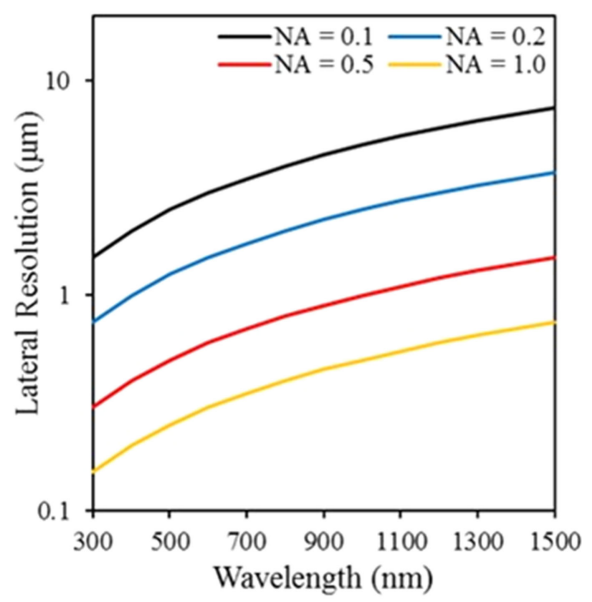

(b)

Fig. 1. OCT systems (a) axial resolution as a function of spectral bandwidth and central wavelength and (b) lateral resolution as a function of numerical aperture and central wavelength, calculated from (1) and (2), respectively.

where $l_{c}$ is axial resolution, or coherence length, $\lambda_{0}$ is the central wavelength and $\Delta \lambda$ is the FWHM. The lateral resolution, which is the minimum diameter of a focused beam, has been taken as

$$
r=\frac{\lambda}{2 n \sin \theta}=\frac{\lambda}{2 N A}
$$

where $r$ is lateral resolution, $n$ is the refractive index of the media, and $\theta$ is the half angle of the light beam that enters the objective. As introduced previously, short wavelength light sources are the most attractive for OCT applications and other optical imaging systems as they offer the potential for improved axial and lateral resolution.

Fig. 1 plots the predicted (a) axial resolution as a function of central wavelength for Gaussian $-3 \mathrm{~dB}$ bandwidths from $5 \mathrm{~nm}$ to $200 \mathrm{~nm}$ using (1). A clear enhancement in resolution by increasing the $-3 \mathrm{~dB}$ bandwidth of the source and by reducing the central wavelength is observed. Fig. 1(b) plots the lateral resolution as a function of central wavelength for numerical apertures from 0.1 to 1.0 using (2). Typical OCT systems have NAs ranging from $\sim 0.2-0.3$ [29], [30]; we can therefore expect in the shift from $1300 \mathrm{~nm}$ to $400 \mathrm{~nm}$, an improvement in lateral resolution from $\sim 5 \mu \mathrm{m}$ to $\sim 1 \mu \mathrm{m}$. However, we do not discuss 
NA and lateral resolution any further in this paper. For obtaining high spectral bandwidth sources, the distribution of carriers within the density of states is critical. If we consider $k_{B} T$ to be proportional to spectral bandwidth, where $k_{B}$ is the Boltzmann coefficient and $T$ is temperature, we find that axial resolution is independent of central wavelength; as in this case $\Delta \lambda$ is equivalent to $\lambda_{0}^{2}\left(k_{\mathrm{B}} / h c\right)$ where $h$ is Planck's constant and $c$ is the speed of light. Hence, lateral resolution enhancement is expected for short wavelength OCT systems even if axial resolution is not enhanced beyond that of near infrared systems $(800,1050$ and $1300 \mathrm{~nm})$ [23].

However, GaN based devices offer a range of differences as compared to GaAs and InP based broad spectral bandwidth devices. GaN materials are robust, with high thermal conductivity and high melting temperatures making them suitable for high current density devices such as SLEDs and semiconductor optical amplifiers. Indium gallium nitride (InGaN) QWs suffer from thickness variations [33], [34] and indium dislocation or clustering [35], [36] causing dot-like behavior [37], resulting in a density of states that lends itself to broad bandwidth applications. Additionally, strong polarization effects (both spontaneous and piezoelectric) result in large inbuilt fields that result in a forward bias significantly modifying the emission wavelength of the device. This, in turn, leads to long carrier lifetimes advantageous for state-filling, which can be exploited by broad bandwidth sources.

Fig. 1 indicates that OCT axial and lateral resolutions can be improved with shorter wavelength light sources. At $\sim 400 \mathrm{~nm}$, sub-cellular axial resolution could be achieved with bandwidths of $\sim 10 \mathrm{~s}$ of $\mathrm{nm}$ and modest NAs.

\section{400 NM OCT SYSTEM}

A $\sim 400 \mathrm{~nm}$ commercial laser diode was modified to operate as a SLED. Utilizing a focused ion beam (FIB) system, a shallow mill (40 nm deep) segmented the ridge contact; removing the top p-contact metal whilst minimizing damage to the waveguide. Areas to either side of the ridge were deep milled $(100 \mathrm{~nm})$, ensuring isolation between the two sections.

Fig. 2(a) shows a scanning electron microscope (SEM) image of the commercial GaN device (Nichia NDV4316) that has been modified to include an $\mathrm{O} / \mathrm{C}$ absorber, hereafter referred to as the FIB-modified commercial device. The total length of the ridge is $\sim 800 \mu \mathrm{m}$ and the length of the $\mathrm{O} / \mathrm{C}$ absorber is $\sim 250 \mu \mathrm{m}$. Optical output power as a function of current density (LJ) is shown in Fig. 2(b). $J_{\text {th }}$ prior to modification (Original $J_{\text {th }}$ ) and after modification (New $J_{\text {th }}$ ) are indicated. $J_{\text {th }}$ shows a significant increase from $\sim 3000$ to $\sim 8100 \mathrm{~A} / \mathrm{cm}^{2}$, with SLED operation (super-linear current-power characteristics) for the current density range between $\sim 4500$ and $\sim 8000 \mathrm{~A} / \mathrm{cm}^{2}$.

Fig. 3 plots normalized emission spectra as a function of wavelength for the commercial laser diode (a) before modification and (b) after modification. As can be seen in Fig. 3(a), before the $\mathrm{O} / \mathrm{C}$ absorber was added, for the laser diode spectra narrowing is observed as current densities increase below lasing threshold $\left(\sim 3000 \mathrm{~A} / \mathrm{cm}^{2}\right)$. After modification, the spectrum remains smooth and continuous with a reasonable bandwidth,
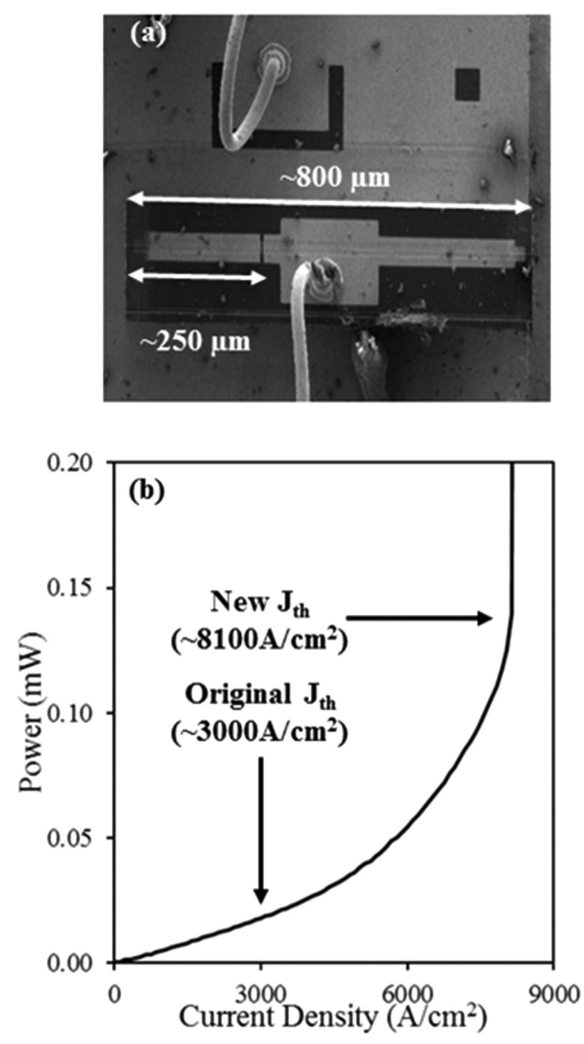

Fig. 2. (a) SEM image of FIB-modified commercial device and (b) modified device LJ characteristics.

which is maintained at current densities much greater than the original $J_{\mathrm{th}}$.

Fig. 4 plots the $-3 \mathrm{~dB}$ bandwidth as a function of current density on the primary axis and the predicted axial resolution from (1) on the secondary axis, for the FIB-modified commercial device. At $\sim 3000 \mathrm{~A} / \mathrm{cm}^{2}$, the original $J_{\mathrm{th}}$, the SLED exhibits a bandwidth $>6 \mathrm{~nm}$, a two-fold increase compared to the unmodified device operating at $\sim 2500 \mathrm{~A} / \mathrm{cm}^{2}$. Equating to $\sim 8 \mu \mathrm{m}$ axial resolution, in line with expectations from Fig. 1(a); this decreases as current density increases until the onset of burn through, and lasing is observed. At lower current densities, a calculated axial resolution of $\sim 5 \mu \mathrm{m}$ is achieved.

Fig. 5 shows the PSFs obtained from the emission spectra shown in Fig. 3(b) for increasing current densities. By inverse Fourier Transforming the emission spectrum, it models an interferometer at zero path length difference, in the case where all the emitted light propagates through the system. By measuring the half-width-half-maximum (HWHM) the theoretical axial resolution can be determined for each current density. As discussed previously, the PSF is used to determine the axial resolution of a light source and is considered more accurate than (1), particularly for highly non-Gaussian emission spectra.

Fig. 6 plots theoretical axial resolution against current density calculated from Fig. 3(b) using (1) and the FWHMs measured from Fig. 5. The difference in calculated resolutions is $<0.5 \mu \mathrm{m}$. Calculated axial resolution can be confirmed experimentally with an interferometer wherein axial resolution is equal to the FWHM of the generated interference pattern. 


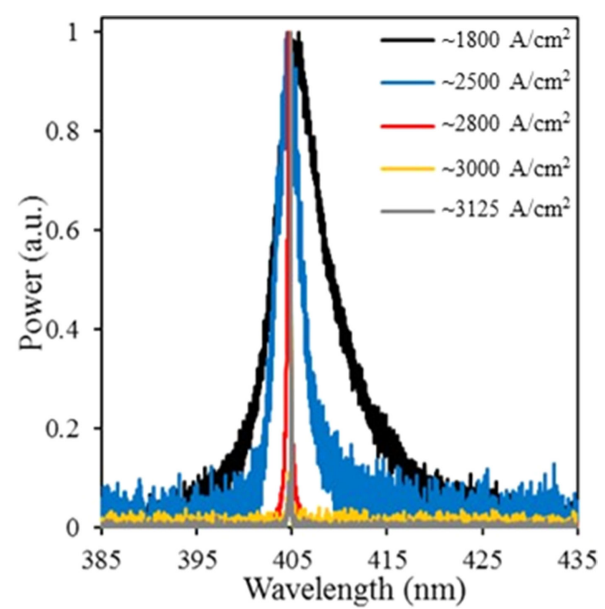

(a)

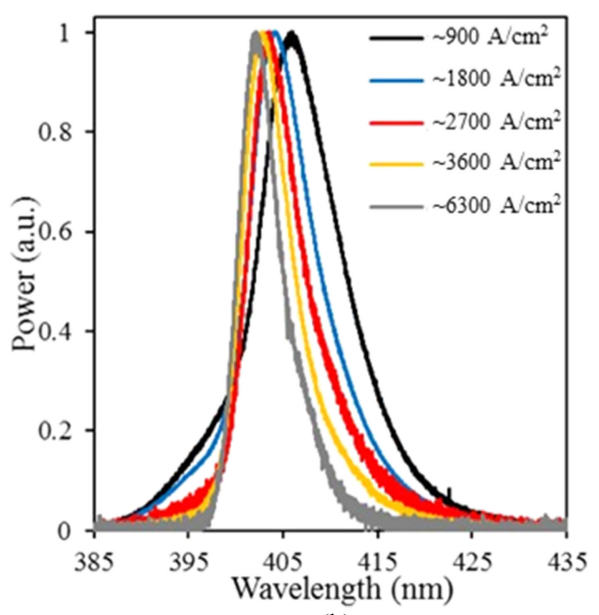

(b)

Fig. 3. Emission spectra of the commercial device with increasing current densities (a) before and (b) after FIB modification.

Fig. 7 shows a schematic of the time-domain OCT system used to generate interference patterns from the commercial GaN device. The divergent light emitted from the device under test (DUT) is collimated by Lens 1 before passing through the 50:50 beam-splitter, where half of the light propagates to the stationary reference mirror while the other half propagates to the scanning mirror. Light reflected from the stationary mirror and the scanning mirror passes through the beam-splitter a second time before it is focused by Lens 2 on to Photodetector 1. By moving the scanning mirror so the path length difference between the 2 beams is $\sim 0$, interference can be observed corresponding to the displacement between the two mirrors. The helium neon (HeNe) laser is aligned with the OCT system allowing displacement of the scanning mirror to be monitored. Replacing the mirror with a multiple layer sample allows layer thickness to be measured [17].

Fig. 8 plots normalized interferograms measured from the FIB-modified commercial device for a range of current densities. The interferograms have been translated vertically, for clarity. With increasing current densities, the $-3 \mathrm{~dB}$ bandwidth of the interferograms increases, increasing the magnitude of axial resolution as predicted in Fig. 4. For $\sim 1800 \mathrm{~A} / \mathrm{cm}^{2}$, the

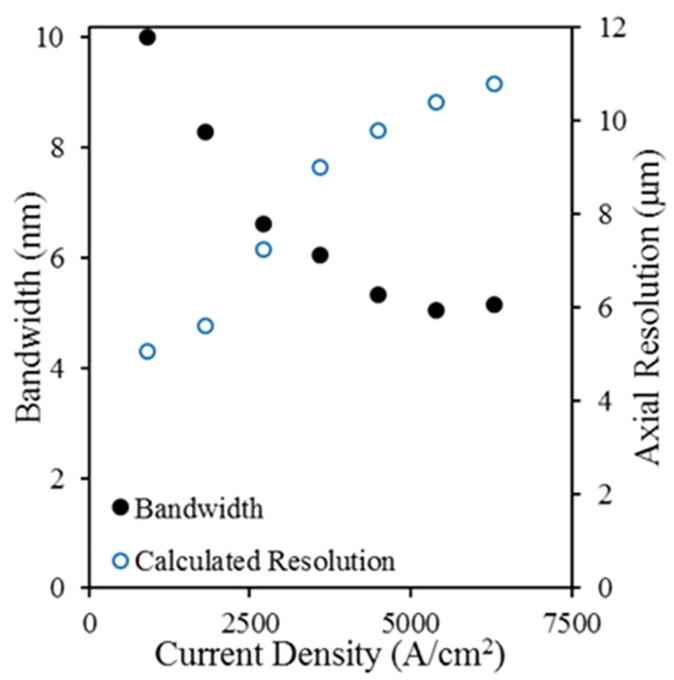

Fig. 4. Spectral bandwidth and calculated axial resolution from (1) for the FIB-modified commercial device as a function of current density.

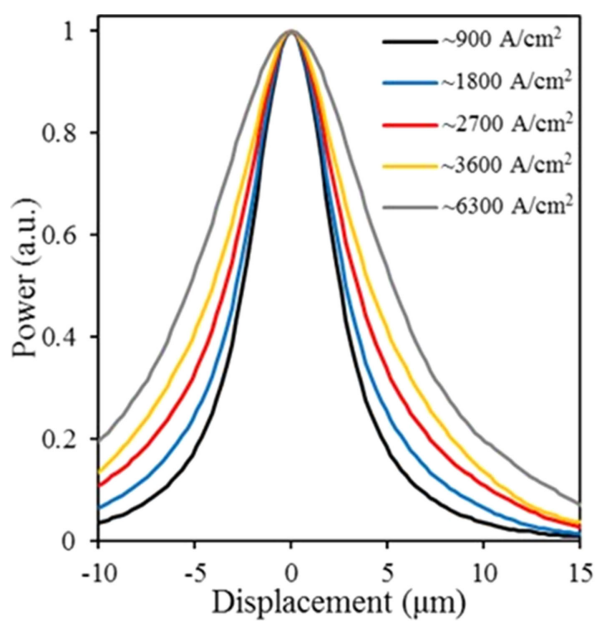

Fig. 5. PSF obtained from the emission spectra of the FIB-modified commercial device for increasing current densities (extracted from Fig. 3(b)).

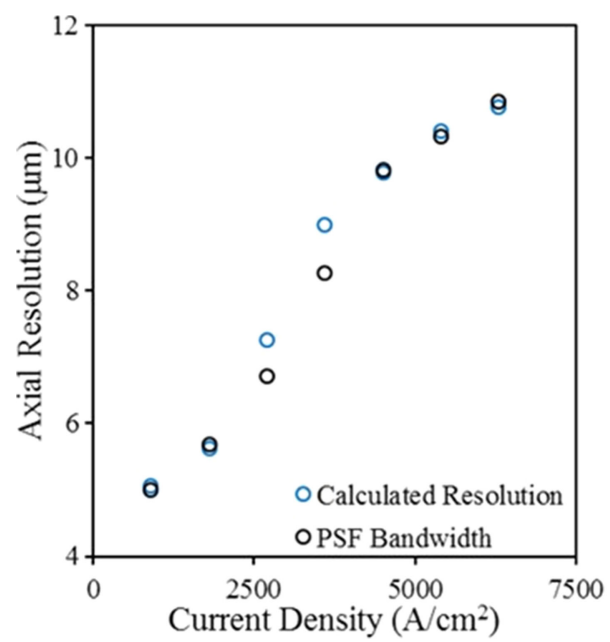

Fig. 6. Comparison of calculated axial resolution from (1) and deducted from the PSF derived from the emission spectrum. 


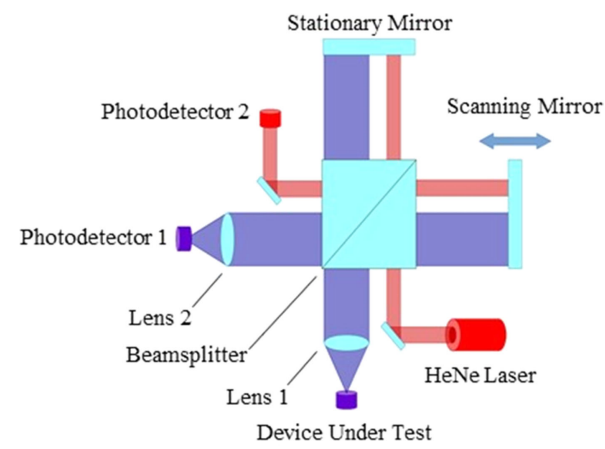

Fig. 7. Schematic of the time-domain OCT system.

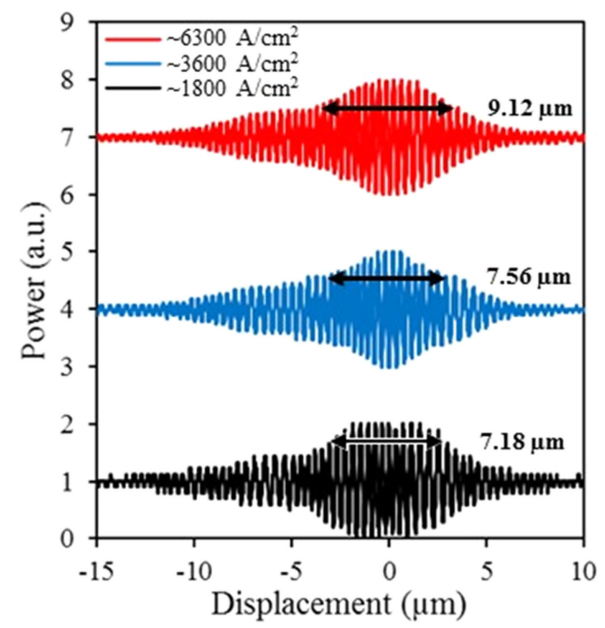

Fig. 8. Interferograms as a function of drive current from FIB-modified commercial device.

predicted axial resolution was $\sim 5.6 \mu \mathrm{m}$, the interference pattern gives axial resolutions of $\sim 7.18 \mu \mathrm{m}$. At $\sim 6300 \mathrm{~A} / \mathrm{cm}^{2}$, the predicted axial resolution is $\sim 10.83 \mu \mathrm{m}$ and measured axial resolution is $\sim 9.12 \mu \mathrm{m}$. We attribute this variation to a combination of system alignment, noise and non-Gaussian nature of the source. At $\sim 3600 \mathrm{~A} / \mathrm{cm}^{2}$ the error between predicted and measured axial resolution is $\sim 0.6 \mu \mathrm{m}$. As such, we note that axial resolution can be closely estimated by the simple Gaussian spectrum approximation (1).

We now demonstrate the operation of the OCT system using the FIB-modified commercial device to measure the thickness of a glass microscope slide.

Fig. 9 plots the normalized interferograms measured from the FIB-modified commercial device when the stationary mirror shown in Fig. 7 was replaced by a microscope slide with a known, nominal thickness of $1100 \mu \mathrm{m}$.

The left interference pattern is produced by light reflecting from the side of the microscope slide nearest the DUT, with light reflecting from the side of the microscope slide furthest from the DUT. The difference between the maxima of interference is $1719 \mu \mathrm{m}$; which, considering the refractive index of the slide, $\sim 1.518$, gives a measured thickness of $\sim 1132 \mu \mathrm{m}$. This demonstrates the interferometer is capable of performing an A-scan [16], albeit in this case with an axial resolution of $\sim 7.5 \mu \mathrm{m}$ (as shown in the inset of Fig. 9), however it shows

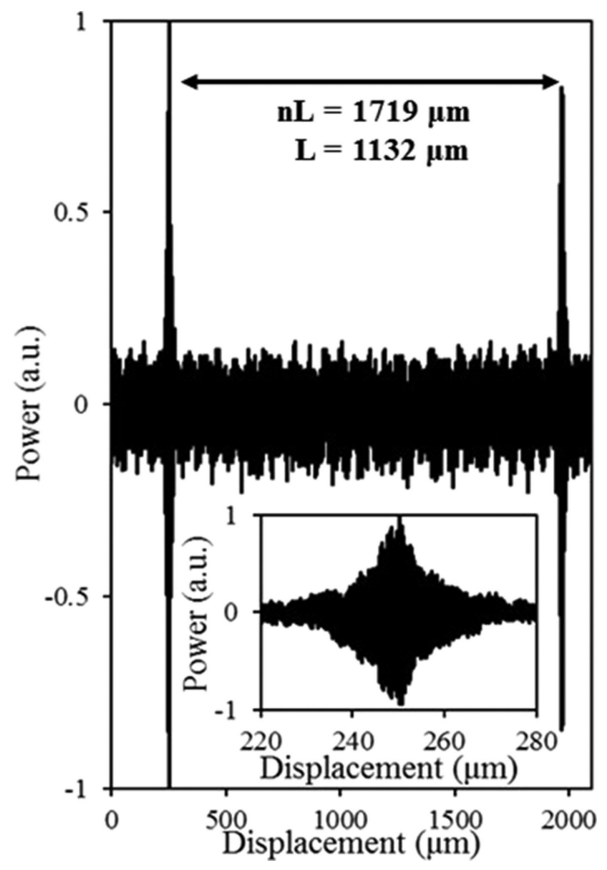

Fig. 9. Interferogram of microscope slide showing thickness measurement with FIB-modified commercial device. Magnified image of the left peak of the interferogram (inset).

proof of concept of the practicality of using $\mathrm{GaN}$ devices for OCT applications. The next step to performing OCT measurements is to use lenses in the system and raster scan the beam.

The low output power of the SLED makes OCT imaging of biological tissue challenging, as the magnitude of the generated interference pattern is indistinguishable from photodetector noise, exacerbated by signal degradation through absorption and scattering [17]-[23]. Output power can be increased by increasing SLED length, as determined from

$$
G_{s}=e\left[\Gamma\left(g_{0} \eta_{i} \frac{J}{d}-\alpha\right) l\right]
$$

where $G_{s}$ is the single pass gain, $\Gamma$ is the confinement factor, $g_{0}$ is the gain coefficient, $\eta_{i}$ is the internal quantum efficiency, $d$ is the active layer thickness in $\mathrm{cm}, \alpha$ is the internal loss and $l$ is the active layer length, in $\mathrm{cm}$ [38]. Fabrication and characterization of longer SLEDs is discussed in Section IV.

\section{BROADBAND GAN SLED}

The SLED in the previous section was fabricated by modifying a commercially available GaN laser. Although the addition of the $\mathrm{O} / \mathrm{C}$ absorber successfully converted the laser into a SLED, the device is far from optimum in terms of output power. Low output power of the light source reduces the detectability of the interferometric signal. Hence, increased output power is required. As demonstrated in (3), output power can be increased by increasing SLED length. In this section we discuss the application of absorbing sections on custom fabricated GaN SLEDs with longer and wider ridge waveguides.

The use of a passive absorber for suppressing lasing in a SLED is well known [9], [25], [26]. However, conventional 


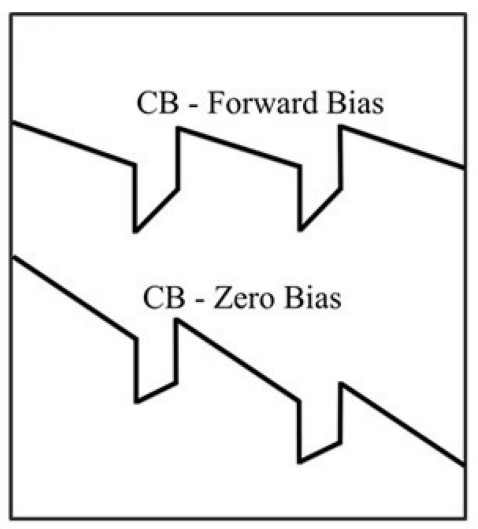

(a)

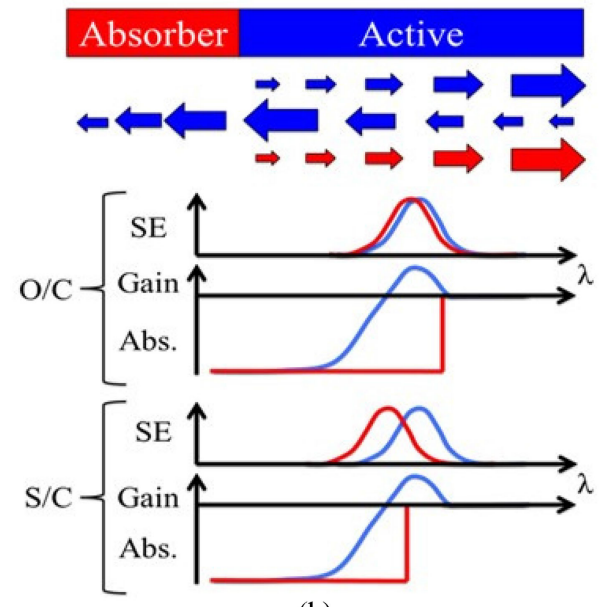

(b)

Fig. 10. Schematic of GaN optical device (a) conduction bands (CBs) under forward and zero bias (b) schematic of SLED made up of absorber and active elements with forward and backward propagating light and recycled (from the absorber) originally backward propagating light. Schematic spontaneous emission, gain spectra of the active and absorber are shown for O/C and S/C operating conditions of the absorber, where $\lambda$ is wavelength.

(0001) GaN based devices differ from conventional (001) GaAs and InP based devices in that strong polarization (piezoelectric and spontaneous) effects induce strong electric fields within the QWs at zero applied bias [39]. For p-i-n structures, due to field-sharing [40], [41], this leads to a forward bias increasing the electric field within the QW [42]; shown schematically in Fig. 10(a).

Fig. 10(b) shows a schematic representation of a SLED device utilizing a forward biased active element and an absorber section that may be $\mathrm{O} / \mathrm{C}$ or S/C. Forward and backward propagating light within the active element is amplified and is either injected into the absorber section or emitted from the device facet. Light injected into the absorber section will be absorbed, and after some time, $t$, be re-emitted as spontaneous emission (SE). A portion of this "recycled" light will propagate along the waveguide, where it experiences gain, towards the front facet. Screening effects due to photo-carriers (and their photo-voltage) are known to play a critical role in determining the emission properties of piezoelectric QW structures [43]. In the case of an $\mathrm{O} / \mathrm{C}$ absorber, at high $\mathrm{J}$, the absorbed backward

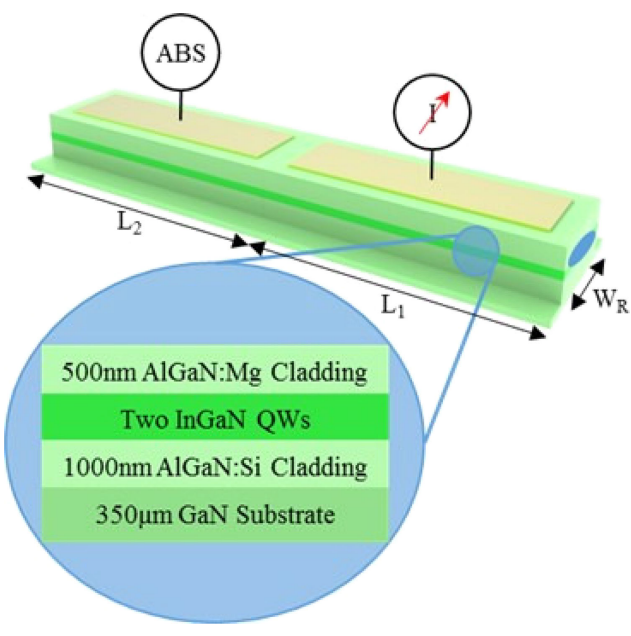

Fig. 11. Schematic of the fabricated multi-section GaN device.

propagating light from the SLED will induce a photo-voltage within the absorber. In GaAs based piezoelectric structures this can come close to the built-in potential of the p-n junction at modest excitation powers [44]. In the case of the $\mathrm{O} / \mathrm{C}$ absorber at low $\mathrm{J}$, and an $\mathrm{S} / \mathrm{C}$ absorber, zero voltage is maintained between the contacts and no photo-voltage is generated. As a result the active element and absorber QWs will have significantly different electric fields, and hence absorption and emission energies. A consequence, for the case of the $\mathrm{O} / \mathrm{C}$ absorber at high $\mathrm{J}$, is almost equal electric fields are expected for the absorber and active elements. Therefore, the bias condition of the absorber is critical in suppressing lasing and preventing burn through. Previous work has shown that a device with an S/C absorber allows higher output powers to be achieved [26]. However, due to the reemission of spontaneous emission back into the active element, the bias condition may also play a key role in enabling broad spectral bandwidth to be realized. Fig. 10(b) illustrates this point, as for an $\mathrm{O} / \mathrm{C}$ absorber at low $\mathrm{J}$, and an $\mathrm{S} / \mathrm{C}$ absorber, the SE from the active element and recycled light will be at different wavelengths that overlap with the gain spectra. However, for an $\mathrm{O} / \mathrm{C}$ absorber at high $\mathrm{J}$, the induced photo-voltage reduces this difference in wavelength and hence reduces the spectral bandwidth. Hakki-Paoli [45] investigations of the commercial device and similar devices indicate similar linewidths and peak positions of the SE and gain spectra.

Fig. 11 shows the schematic of the $\sim 2600 \mu \mathrm{m}$ long multisection GaN SLED, where $\mathrm{W}_{\mathrm{R}}$ is the ridge width $(10 \mu \mathrm{m}), \mathrm{L}_{1}$ is the front pumped section length $(\sim 1750 \mu \mathrm{m})$ and $\mathrm{L}_{2}$ is the rear absorber (ABS) section length $(\sim 850 \mu \mathrm{m})$.

Devices were fabricated from an epitaxial wafer with 2 InGaN QWs grown by metalorganic vapor phase epitaxy on a 2 inch GaN substrate by Novagan [10], [12]; the SLED features a straight waveguide perpendicular to both facets and no anti-reflection or high reflection coatings. Lasing is therefore suppressed only by the absorber section, which can be operated either $\mathrm{O} / \mathrm{C}$ or $\mathrm{S} / \mathrm{C}$.

The devices were fabricated using standard contact photolithography and the ridge waveguides were etched below 


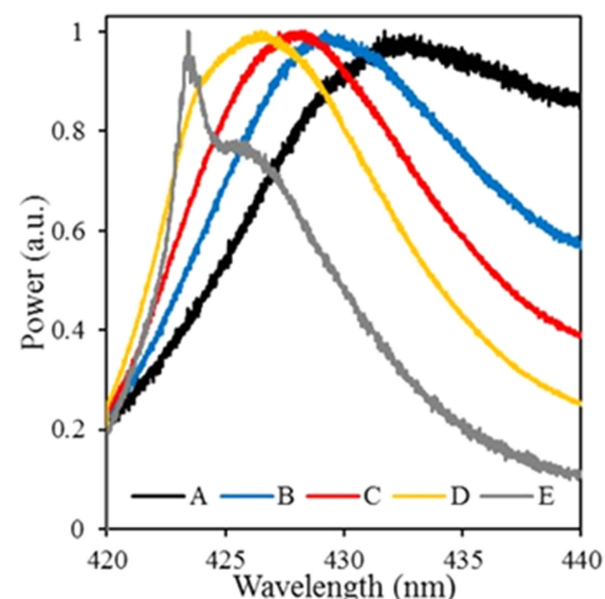

(a)

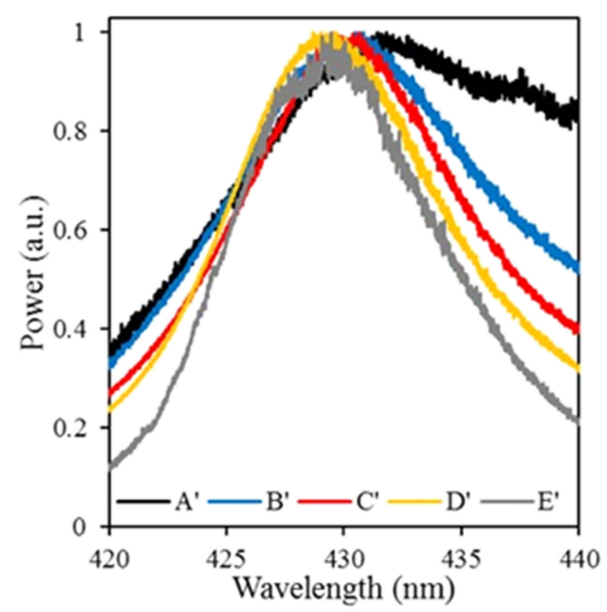

(b)

Fig. 12. LJ characteristics for the $\mathrm{O} / \mathrm{C}$ and $\mathrm{S} / \mathrm{C}$ absorber for the fabricated $\mathrm{GaN}$ device.

the QW region using a plasma-enhanced chemical vapor deposition (PECVD) silicon dioxide mask and an inductively coupled plasma reactive-ion etch process, using $\mathrm{SiCl}_{4} / \mathrm{Cl}_{2} / \mathrm{Ar}$ gases. The isolation etches between the sections were similarly etched, but to a depth above the active region. A $300 \mathrm{~nm}$ PECVD silicon dioxide layer was deposited as an insulating layer, and an ohmic Ni/Au top contact and Ti/Au bondpad were deposited by thermal evaporation. A thermally evaporated Ti/Al/Ti/Au contact was deposited for the $\mathrm{n}$-contact.

Fig. 12 plots the LJ curve for the SLED when the absorber is operated in $\mathrm{O} / \mathrm{C}$ (black) and $\mathrm{S} / \mathrm{C}$ (blue) under pulsed conditions. With the $\mathrm{O} / \mathrm{C}$ absorber, output power $>25 \mathrm{~mW}$ is observed, but as with the device in Section II, burn through is observed at $J_{\text {th }} \geq 7000 \mathrm{~A} / \mathrm{cm}^{2}$. For the $\mathrm{S} / \mathrm{C}$ absorber, an output power $\sim 70 \mathrm{~mW}$ was achieved with no evidence of burn through, up to current densities of $>15000 \mathrm{~A} / \mathrm{cm}^{2}$.

Fig. 13 plots the normalized SLED emission spectra from 420-440 nm under pulsed operation for a range of injected current densities with the absorber (a) $\mathrm{O} / \mathrm{C}$ where $\mathrm{A}, \mathrm{B}, \mathrm{C}$, $\mathrm{D}, \mathrm{E}$ are $\sim 1500, \sim 3000, \sim 4500, \sim 6000$, and $\sim 7500 \mathrm{~A} / \mathrm{cm}^{2}$; respectively and (b) S/C, where A', B', C', D', E' are $\sim 1500$, $\sim 3000, \sim 4500, \sim 6000$, and $\sim 15000 \mathrm{~A} / \mathrm{cm}^{2}$, respectively. A

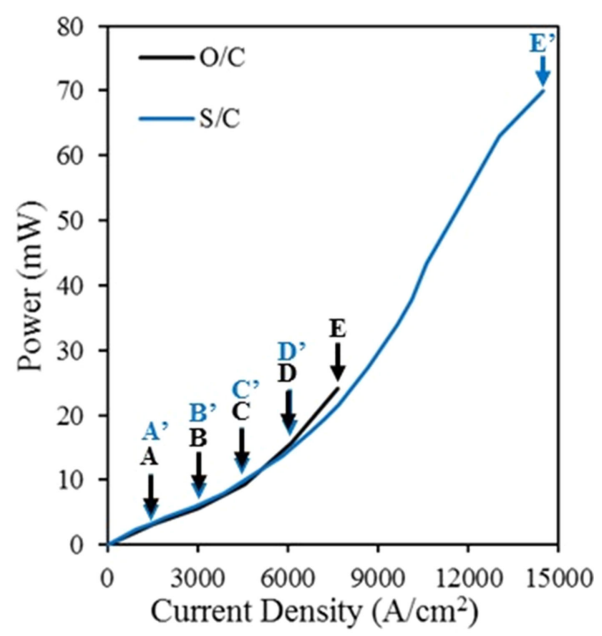

Fig. 13. Emission spectra with the absorber (a) O/C and (b) S/C for the fabricated $\mathrm{GaN}$ device.

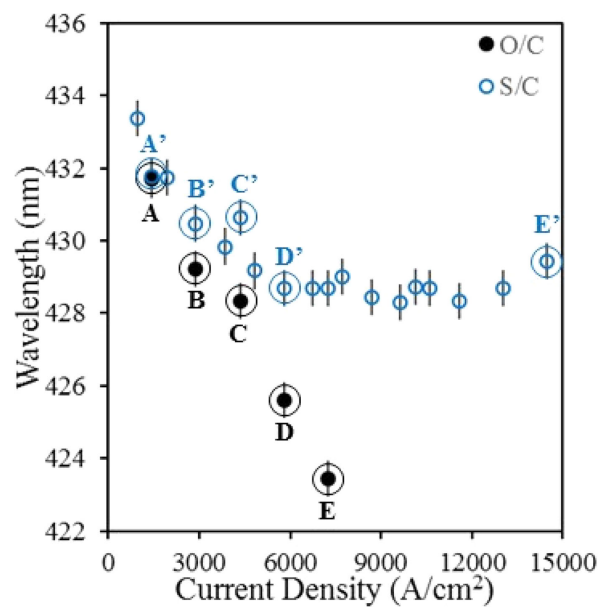

Fig. 14. Central wavelength as a function of current density for $\mathrm{O} / \mathrm{C}$ and $\mathrm{S} / \mathrm{C}$ absorber for the fabricated GaN device.

lasing peak is clearly observed in Fig. 13(a), but is not observed for any investigated biases in Fig. 13(b). Operating the SLED with an S/C absorber yields a wider emission spectrum than an $\mathrm{O} / \mathrm{C}$ absorber, with the bandwidth at $\sim 30 \mathrm{~nm}$ for low current densities, decreasing to $10 \mathrm{~nm}$ for current densities between $\sim 6000-15000 \mathrm{~A} / \mathrm{cm}^{2}$.

Fig. 14 plots the peak emission wavelength against current density and Fig. 15 plots the $-3 \mathrm{~dB}$ bandwidth against current density with the absorber $\mathrm{O} / \mathrm{C}$ and S/C. These dependencies have been extracted from the spectra shown in Fig. 13(a) and (b), with circles highlighting which point corresponds to which emission spectrum. With an $\mathrm{O} / \mathrm{C}$ absorber there is a shift in the central emission wavelength from 432 to $423 \mathrm{~nm}$ as current density increases. With the absorber S/C peak wavelength shifts from 433 to $428 \mathrm{~nm}$ over the same current range.

Fig. 16 plots the HWHM of the PSF of the emission spectra of fabricated $\mathrm{GaN}$ device as a function of current density with the absorber $\mathrm{O} / \mathrm{C}$ and $\mathrm{S} / \mathrm{C}$. This value provides an estimate of the axial resolution obtainable in an OCT system. However, we 


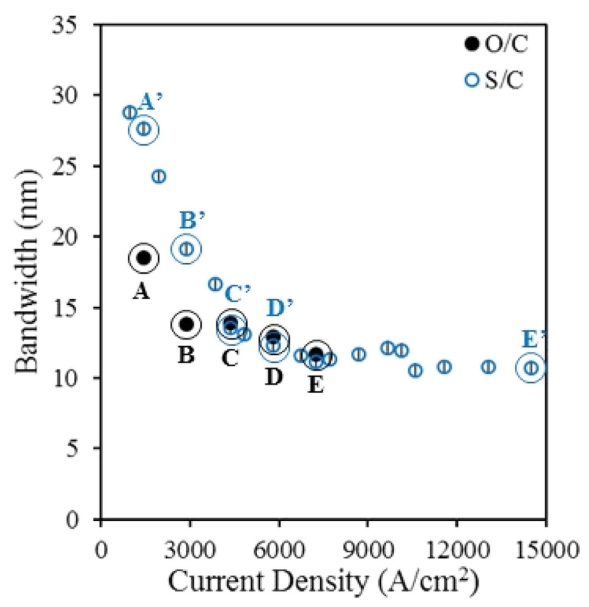

Fig. 15. $-3 \mathrm{~dB}$ bandwidth as a function of current density for $\mathrm{O} / \mathrm{C}$ and $\mathrm{S} / \mathrm{C}$ absorber for the fabricated $\mathrm{GaN}$ device.

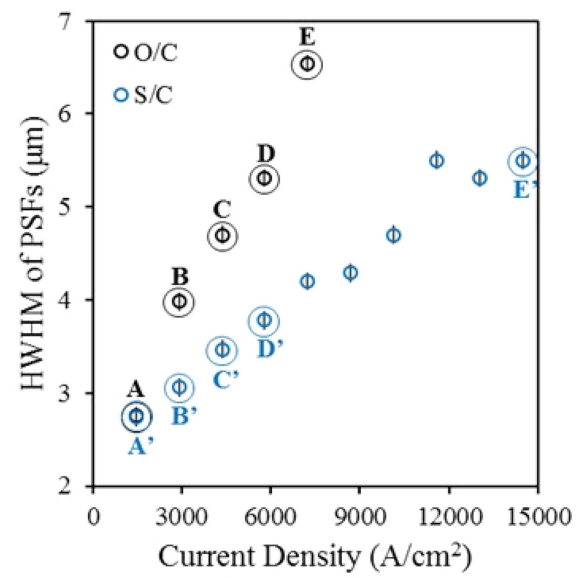

(a)

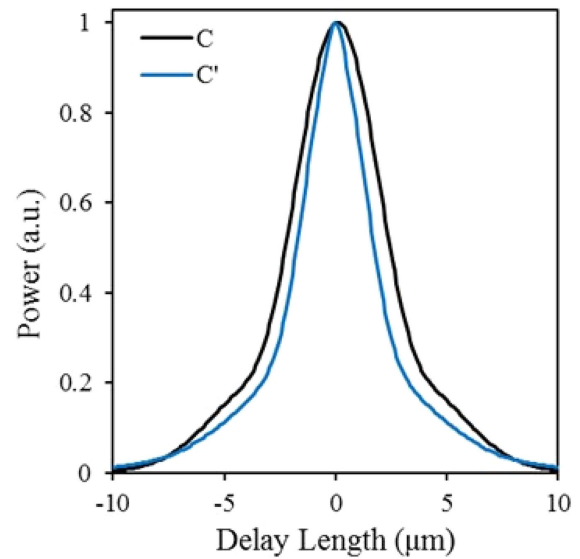

(b)

Fig. 16. (a) Predicted axial resolution as a function of current density obtained from the HWHM of the PSFs for O/C and S/C absorber for the GaN device (b) PSFs for $\mathrm{C}(\mathrm{O} / \mathrm{C})$ and $\mathrm{C}^{\prime}(\mathrm{S} / \mathrm{C})$.

note that this may be overoptimistic of the achievable resolution as any additional spectral components of the emission spectrum beyond a single Gaussian will result in power to large positive and negative delays within the coherence function. This may result in ghost images or a resolution penalty due to spectral modulation [46]. At low current densities, the axial resolution is almost identical whether the absorber is $\mathrm{O} / \mathrm{C}$ or $\mathrm{S} / \mathrm{C}$ (i.e., in the limit of zero photo-voltage applied to the absorber), with an HWHM of $\sim 2.74 \mu \mathrm{m}$. As the current density increases, the axial resolution decreases at a faster rate for the $\mathrm{O} / \mathrm{C}$ absorber, even for a $9 \mathrm{~nm}$ blue-shift in central emission wavelength counteracting the reduction in bandwidth for the $\mathrm{O} / \mathrm{C}$ emission spectrum. For the $\mathrm{S} / \mathrm{C}$ absorber, after the initial reduction, an $\mathrm{HWHM} \sim 5.5 \mu \mathrm{m}$ for all current densities investigated suggesting it would be a suitable device for an OCT system.

\section{CONCLUSION}

In summary, the role of the $-3 \mathrm{~dB}$ bandwidth, central wavelength and NA on the axial and lateral resolutions of OCT systems has been explored. Opportunities for sub-cellular resolution imaging using broadband $\mathrm{GaN}$ based light sources have been discussed. We have shown that a commercial GaN laser can be modified to produce a SLED, and employed in a $\sim 400 \mathrm{~nm}$ time-domain OCT system. Different methods to determine OCT system axial resolution have been compared and metrology measurements performed on a transparent sample. Finally, the effect of piezoelectric and spontaneous polarization in GaN devices has been discussed and shown experimentally, on bespoke GaN SLEDs, and the absorber bias condition has been shown to play a critical role in GaN SLED bandwidths. Such devices lend themselves to $\sim 3-4 \mu \mathrm{m}$ axial resolution OCT imaging.

\section{ACKNOWLEDGMENT}

The authors gratefully acknowledge assistance from G. Li in figure preparation.

\section{REFERENCES}

[1] S. Nakamura, T. Mukai, and M. Senoh, "High-power GaN P-N junction blue-light-emitting diodes," Jpn. J. Appl. Phys., vol. 30, no. 12A, pp. L1998-L2001, Dec. 1991.

[2] S. Nakamura et al., "InGaN-Based multi-quantum-well-structure laser diodes," Jpn. J. Appl. Phys., vol. 35, pp. L74-L76, Jan. 1996.

[3] S. Nakamura, M. Senoh, and T. Mukai, "P-GaN/N-InGaN/N-GaN doubleheterostructure blue-light-emitting diodes," Jpn. J. Appl. Phys., vol. 32, pp. L8-L11, Jan. 1993.

[4] S. Nakamura, M. Senoh, and T. Mukai, "High-power InGaN/GaN doubleheterostructure violet light emitting diodes," Appl. Phys. Lett., vol. 62, pp. 2390-2392, May 1993.

[5] S. Nakamura et al., "Continuous-wave operation of InGaN multiquantum-well-structure laser diodes at 233K," Appl. Phys. Lett., vol. 69, pp. 3034-3036, Nov. 1996.

[6] S. Nakamura et al., "High-power, long-lifetime InGaN multi-quantumwell-structure laser diodes," Jpn. J. Appl. Phys., vol. 36, pp. L1059-1061, Aug. 1997.

[7] T. Miyoshi et al., "Recent status of white LEDs and nitride LDs," Proc. SPIE, vol. 6894, pp. 689414-1-689414-7, Feb. 2008.

[8] Y. Kasami et al., "Large capacity and high-data-rate phase-change disks," Jpn. J. Appl. Phys., vol. 39, pp. 756-761, Feb. 2000.

[9] A. Kafar et al., "Cavity suppression in nitride based superluminescent diodes," J. Appl. Phys., vol. 111, Apr. 2012, Art. no. 083106.

[10] E. Feltin et al., "Broadband blue superluminescent light-emitting diodes based on GaN," Appl. Phys. Lett., vol. 95, Aug. 2009, Art. no. 081107.

[11] W. O. Davis, R. Sprague, and J. Miller, "MEMS-based pico projector display," in Proc. IEEE/LEOS MEMS Nanophot., Aug. 2008, pp. 31-32.

[12] M. Rossetti et al., "High power blue-violet superluminescent light emitting diodes with InGaN quantum wells," Appl. Phys. Express, vol. 3, May 2010, Art. no. 061002. 
[13] F. Kopp et al., "Blue superluminescent light-emitting diodes with output power above $100 \mathrm{~mW}$ for picoprojection," Jpn. J. Appl. Phys., vol. 52, May 2013, Art. no. 08JH07.

[14] H. Ohno, K. Orita, M. Kawaguchi, K. Yamanaka, and S. Takigawa, "200 mW GaN-based superluminescent diode with a novel waveguide structure," in Proc. IEEE Photon. Soc. 24th Annu. Meeting, Oct. 2011, pp. 505-506.

[15] A. Kafar et al., "High-optical-power InGaN superluminescent diodes with "j-shape" waveguide," Appl. Phys. Express, vol. 6, Aug. 2013, Art. no. 092102.

[16] D. Huang et al., "Optical coherence tomography," Science, vol. 254, pp. 1178-1181, Sep. 1991.

[17] H. Shibata et al., "Imaging of spectral-domain optical coherence tomography using a superluminescent diode based on InAs quantum dots emitting broadband spectrum with gaussian-like shape," Jpn. J. Appl. Phys., vol. 54, Mar. 2015, Art. no. 04DG07.

[18] J. A. Izatt, M. D. Kulkarni, H.-W. Wang, K. Kenji, and M. V. Sivak Jr., "Optical coherence microscopy in gastrointestinal tissues," IEEE J. Sel. Topics Quantum Electron., vol. 2, no. 4, pp. 1017-1028, Dec. 1996.

[19] A. D. Aguirre, P. Hsiung, T. H. Ko, I. Hartl, and J. G. Fujimoto, "Highresolution optical coherence microscopy for high-speed, in vivo cellular imaging," Opt. Lett., vol. 28, no. 21, pp. 2064-2066, Nov. 2003.

[20] W. Drexler et al., "Ultrahigh-resolution ophthalmic optical coherence tomography," Nature Med., vol. 7, no. 4, pp. 502-507, Apr. 2001.

[21] A. Unterhuber et al., "Advances in broad bandwidth light sources for ultrahigh resolution optical coherence tomography," Phys. Med. Biol., vol. 49, pp. 1235-1246, Mar. 2004.

[22] H. Hirayama and S. Nakamura, "Development of ultraviolet- and visiblelight one-shot spectral domain optical coherence tomography and in situ measurements of human skin," J. Biomed. Opt., vol. 20, Jul. 2015, Art. no. 076014

[23] S. Maliszewska and M. Wojtkowski, "Broadband blue light for optical coherence microscopy," Photon. Lett. Poland, vol. 3, pp. 138-140, Dec. 2011

[24] V. R. Shidlovski, Optical Coherence Tomography, 2nd ed., W. Drexler and J. G. Fujimoto, Eds. Cham, Switzerland: Springer, 2015, pp. 505-526.

[25] A. Kafar et al., "Design and optimization of InGaN superluminescent diodes," Phys. Status Solidi A, vol. 212, no. 5, pp. 997-1004, Jan. 2015.

[26] N. S. K. Kwong, K. Y. Lau, and N. Bar-Chaim, "High-power highefficiency GaAlAs superluminescent diodes with an internal absorber for lasing suppression," IEEE J. Quantum Electron., vol. 25, no. 4, pp. 696-704, Apr. 1989.

[27] A. Kafar et al., "Nitride superluminescent diodes with broadened emission spectrum fabricated using laterally patterned substrate," Opt. Express, vol. 24, no. 9, pp. 9673-9682, Apr. 2016

[28] A. Kafar et al., "InAlGaN superluminscent diodes fabricated on patterned substrates: an alternative semiconductor broadband emitter," Photon. Res., vol. 5, no. 2, pp. A30-A34, Apr. 2017.

[29] C. Akcay, P. Parrein, and J. P. Rolland, "Estimation of longitudinal resolution in optical coherence imaging," Appl. Opt., vol. 41, no. 25, pp. 5256-5262, Sep. 2002.

[30] A. C. Akcay, J. P. Rolland, and J. M. Eichenholz, "Spectral shaping to improve the point spread function in optical coherence tomography," Opt. Lett., vol. 28, no. 20, pp. 1921-1923, Oct. 2003.

[31] W. Drexler, "Ultrahigh-resolution optical coherence tomography," $J$. Biomed. Opt., vol. 9, no. 1, pp. 47-74, Jan. 2004

[32] Z. Ding, H. Ren, Y. Zhao, J. S. Nelson, and Z. Chen, "High-resolution optical coherence tomography over a large depth range with an axicon lens," Opt. Lett., vol. 27, no. 4, pp. 243-245, Feb. 2002.

[33] A. B. Yankovich et al., "Thickness variations and absence of lateral compositional fluctuations in aberration-corrected STEM images of InGaN LED active regions at low dose," Microsc. Microanal., vol. 20, pp. 864-868, Feb. 2014. [Online]. Available: https://www.cambridge.org/ core/services/aop-cambridge-core/content/view/A89ADEF5A2BE5483 58E440C0B272D02F/S1431927614000427a.pdf/thickness_variations_ and_absence_of_lateral_compositional_fluctuations_in_aberration corrected_stem_images_of_ingan_led_active_regions_at_low_dose.pdf

[34] C. G. Park, G. H. Gu, B. H. Lee, and D. H. Jang, "Effects of growth pressure on the structural and optical properties of multi quantum wells (MQWs) in blue LED," Ultramicroscopy, vol. 127, pp. 114-118, Jul. 2013.
[35] H. K. Cho et al., "Effect of growth interruptions on the light emission and indium clustering of InGaN/GaN multiple quantum wells," Appl. Phys. Lett., vol. 79, no. 16, pp. 2594-2596, Oct. 2001.

[36] A. Rosenauer et al., "Composition mapping in InGaN by scanning transmission electron microscopy," Ultramicroscopy, vol. 111, pp. 1316-1327, Apr. 2011.

[37] K. Tachibana, T. Someya, and Y. Arakawa, "Nanometer-Scale InGaN Self-assembled quantum dots grown by metalorganic chemical vapor deposition,” Appl. Phys. Lett., vol. 74, no. 3, pp. 383-385, Jan. 1999.

[38] G. A. Alphonse, D. B. Gilbert, M. G. Harvey, and M. Ettenberg, "Highpower superluminescent diodes," IEEE J. Quantum Electron., vol. 24, no. 12 , pp. $2454-2457$, Dec. 1988

[39] T. Takeuchi et al., "Quantum-confined stark effect due to piezoelectric fields in GaInN strained quantum wells," Jpn. J. Appl. Phys., vol. 36, no. 4A, pp. L382-L385, Apr. 1997.

[40] A. S. Pabla et al., "Partial screening of internal electric fields in strained piezoelectric quantum well lasers: implications for optoelectronic integration," Appl. Phys. Lett., vol. 68, pp. 1595-1597, Jan. 1996.

[41] R. A. Hogg et al., "Piezoelectric-field effects on transition energies, oscillator strengths, and level widths in (111)B-grown (In,Ga)As/GaAs multiple quantum wells," Phys. Rev. B, vol. 48, no. 11, pp. 8491-8494, Sep. 1993.

[42] T. Takeuchi et al., "Determination of piezoelectric fields in strained GaInN quantum wells using the quantum-confined stark effect," Appl. Phys. Lett., vol. 73, no. 12, pp. 1691-1693.

[43] T. E. Sale et al., "Carrier screening effects in piezoelectric strained InGaAs/GaAs quantum wells grown on the [111]B axis," J. Appl. Phys., vol. 76, no. 9, pp. 5447-5452, Jul. 1994.

[44] J. P. R. David et al., "Excitation power and barrier width dependence of photoluminescence in piezoelectric multiquantum well p-i-n structures," Appl. Phys. Lett., vol. 68, pp. 820-822, Nov. 1995.

[45] B. W. Hakki and T. L. Paoli, "Gain spectra in GaAs double-heterostructure injection lasers," J. Appl. Phys., vol. 46, no. 3, pp. 1299-1306, Mar. 1975.

[46] S. Chen et al., "GaAs-Based superluminescent light-emitting diodes with 290-nm emission bandwidth by using hybrid quantum well/quantum dot structures," Nanoscale Res. Lett., vol. 10, pp. 1-8, Aug. 2015.

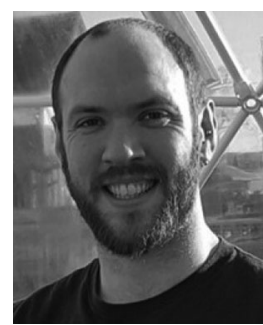

Graham R. Goldberg received the B.Eng. degree in electrical engineering and the M.Phil. degree in electronic engineering from the Electronic and Electrical Engineering Department, The University of Sheffield, Sheffield, U.K., in 2010 and 2014, respectively. He is currently working toward the Ph.D. degree in gallium nitride optical devices as part of the Photonic Devices and Systems Group, Department of Electronic and Nanoscale Engineering, School of Engineering, The University of Glasgow, Glasgow, U.K.

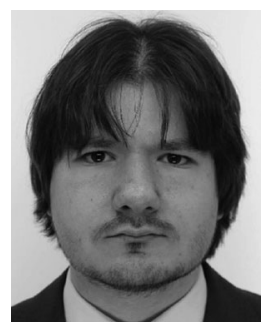

Aleksandr Boldin received the B.Eng. degree in electronic engineering from the Electronic and Electrical Engineering Department, The University of Sheffield, Sheffield, U.K., in 2017. He is currently completing an engineering placement at The University of Sheffield prior to working toward the Ph.D. degree in vertical cavity surface emitting lasers as part of the Photonic Devices and Systems Group, Department of Electronic and Nanoscale Engineering, The University of Glasgow, Glasgow, U.K. from September 2017 


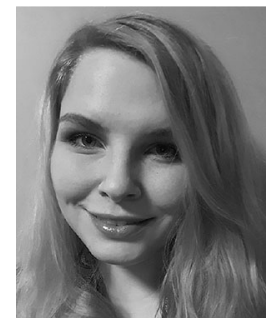

Sophia M. L. Andersson received the M.Sci. degree in physics from the School of Physics and Astronomy, The University of Glasgow, Glasgow, U.K., in 2016, where she also worked as part of the Materials and Condensed Matter Physics Group and was the co-author on two publications. She is currently working toward the Ph.D. degree in photonic integration and advanced data storage with the Centre for Doctoral Training, a partnership between The University of Glasgow, Glasgow, U.K., and Queen's University Belfast, Belfast, U.K.

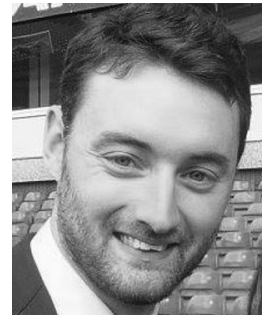

Richard J. E. Taylor received the B.Eng. degree in electrical engineering, the M.Sc. degree in semiconductor photonics, and the Ph.D. degree in photonic crystal surface emitting lasers, from the Electronic and Electrical Department, The University of Sheffield, Sheffield, U.K., in 2010, 2011, and 2015, respectively. Upon completion of the Ph.D. degree, he was awarded the EPSRC doctoral prize fellowship at the University of Sheffield.

$\mathrm{He}$ is currently a JSPS Postdoctoral Research Fellow at the University of Tokyo, Tokyo, Japan.

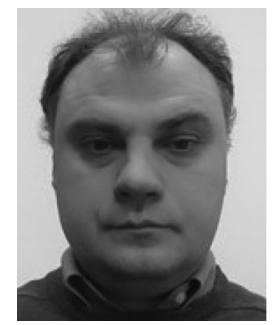

Pavlo Ivanov received the Dipl.-Ing. degree in electrical engineering (with Honors) and the Ph.D. degree in optics and laser physics, both from the Kharkiv National University of Radio and Electronics, Kharkiv, Ukraine, in 1999 and 2004, respectively.

In 2003-2011, he was a Visiting Fellow, Research Associate, and a GWR Research Fellow at the University of Bristol, Bristol, U.K. During this time, he was involved in theoretical and experimental investigation of photonic crystal displays and VCSELs incorporating two-dimensional photonic crystals. In 2012-2013, he was a Research Associate at the University of Leeds, Leeds, U.K., working on modelling of SiGe quantum cascade lasers. In 2013-2015, he became a Research Associate at the University of Sheffield, Sheffield, U.K., and in 2015, he joined the University of Glasgow, Glasgow, U.K. His current research interests include the theory and modelling of semiconductor light-emitting devices, high-performance computing, postprocessing of devices using focused ion beam systems, experimental study of laser device characteristics and optical systems.

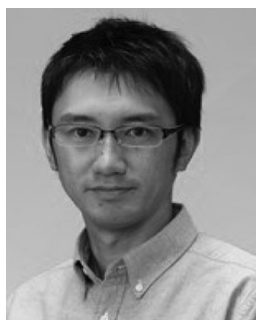

Nobuhiko Ozaki received the B.Sc., M.Sc., and Ph.D. degrees in physics from Osaka University, Japan, in 1997, 1999, and 2002, respectively.

In 2002, he joined the Institute of Materials Science, University of Tsukuba, Japan, as a Research Associate. He was engaged in the development of the ferromagnetic semiconductor, $(\mathrm{Zn}, \mathrm{Cr}) \mathrm{Te}$, for spintronic device applications. In 2005, he joined the Center for Tsukuba Advanced Research Alliance, University of Tsukuba, Japan, as an Assistant Professor, where he worked on the development of all-optical signal processing devices based on photonic crystal waveguides and quantum dots. In 2009, he moved to the Faculty of Systems Engineering, Wakayama University, Japan, as an Associate Professor. From 2014 to 2015, he was a Visiting Academic in the Department of Electronic and Electrical Engineering, The University of Sheffield, Sheffield, U.K. His current research includes the selective-area-growth of quantum dots and their application to near-infrared broadband light sources for optical coherence tomography applications. He is the author or co-author of 65 refereed papers, 36 conference proceedings, and 4 book chapters.

Dr. Ozaki is a member of the Japan Society of Applied Physics and the Institute of Electronics, Information and Communication. He received the " $18^{\text {th }}$ Young Scientist Award for the Presentation of an Excellent Paper," awarded by JSAP in 2005 and the " $20^{\text {th }}$ Ando Incentive Prize for the Study of Electronics," awarded by the Foundation of Ando Laboratory in 2007.

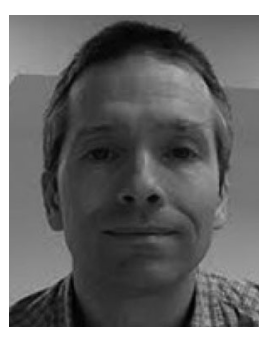

David T. D. Childs (M'15) received the B.Sc. degree in physics and the M.Sc. degree in semiconductor science and technology from the Imperial College, London, U.K., in 1996 and 1997, respectively, and the Ph.D. degree in properties and applications of InAs/GaAs quantum dot devices from the Imperial College in 2002.

He was then with the R\&D Department of Marconi Optical Components (later Bookham now Oclaro) at the Caswell semiconductor research facility until 2006, where he was responsible for the development of a range of telecoms lasers. During this time he also worked on several European projects developing quantum dot technology. Following this he joined the Department of Electronic and Electrical Engineering, The University of Sheffield. There he was engaged in a number of projects developing semiconductor light sources from visible through to $\mathrm{THz}$ wavelengths. He was also involved in developing systems to demonstrate the application of semiconductor devices to fields ranging from selective laser melting (3-D printing), to mid-infrared hyper-spectral imaging (biomedical imaging). Since 2015, he has been a Lecturer in the Department of Electronic and Nanoscale Engineering, School of Engineering, The University of Glasgow, Glasgow, U.K. His research interests span from semiconductor light emitter development through photonic integration to the applications of these devices and systems from communications to biomedicine. He has contributed to more than 100 journal and conference publications.

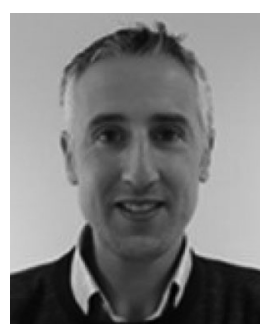

Kristian M. Groom (M'03) received the M.Phys. and Ph.D. degrees from the Department of Physics and Astronomy, The University of Sheffield, Sheffield, U.K., in 1999 and 2003, respectively.

During 2005-2010, he was a Royal Academy of Engineering Research Fellow, with research concentrated on advanced GaAs optoelectronic components based upon a buried waveguide technology, including self-aligned stripe lasers, DFB lasers, superluminescent diodes, optical amplifiers, and surface emitting lasers. He is currently a Senior Lecturer in the Department of Electronic and Electrical Engineering, The University of Sheffield. His current research interests include design and manufacture of a range of optoelectronic components and integrated circuits and their ability to provide solutions in emerging applications, such as those in advanced manufacturing. He has authored or co-authored more than 60 refereed journal articles and has an h-index of 20. 


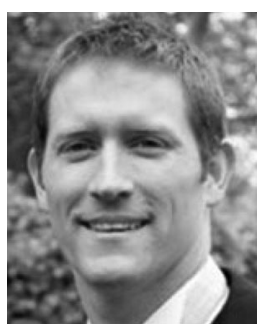

Kenneth L. Kennedy received the B.Sc. degree in optoelectronics from Heriot-Watt University, Edinburgh, U.K., in 1999 and the Ph.D. degree in electronic and electrical engineering from the University of Sheffield, Sheffield, U.K., in 2009. After graduating he was awarded with a Postdoctoral Prize Fellowship, funded by the EPSRC, working mainly on quantum cascade laser technologies. He immediately joined Hewlett Packard, Ipswich, U.K., as a Manufacturing/R\&D Engineer working on telecom wavelength emitters and detectors. In 2004, he joined Agilent Technologies Ltd, Singapore, as a Technology Transfer Engineer.

He currently works for the EPSRC National Centre for III-V technologies, The University of Sheffield, Sheffield, U.K., and is responsible for the device fabrication of a variety of devices and materials. His research interests include semiconductor laser and emitter technology.

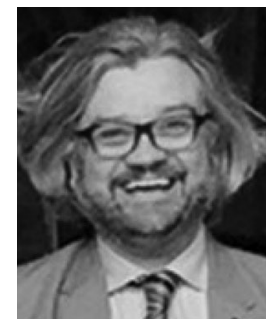

Richard A. Hogg received the Ph.D. degree from the Department of Physics and Astronomy, The University of Sheffield, Sheffield, U.K., in 1995, and then spent two years as a Postdoctoral Researcher at NTT Basic Research Laboratories, Atsugi, Japan.

He was then awarded an EU-Japan fellowship as a Visiting Researcher in Professor Arakawa's Laboratory, University of Tokyo, Tokyo, Japan. He subsequently spent three years at Toshiba Research Europe's Cambridge Laboratory, before moving to Agilent Technologies Fibre-Optic Component Operation in Ipswich, U.K., in 2000. In 2003, he joined the Electronic and Electrica Engineering Department, The University of Sheffield, Sheffield, U.K. Since 2015, he has been a Professor of electronic and nanoscale engineering at the University of Glasgow, Glasgow, U.K. His research group is active in developing the understanding of device physics and engineering, epitaxial processes, fabrication technologies and applications of various semiconductor laser, amplifier, and superluminescent light emitting diode devices. 